\title{
Potentially prothrombotic abnormalities of coagulation in benign intracranial hypertension
}

\author{
J Sussman, M Leach, M Greaves, R Malia, G A B Davies-Jones
}

\begin{abstract}
Objective-Benign intracranial hypertension (BIH) may be caused by intracranial venous sinus thrombosis. Cerebral angiograms may, however, be normal in patients with BIH that are associated with conditions with an increased risk of venous thrombosis. This raises the possibility that unrecognised non-occlusive venous thrombus might impede CSF drainage. This study therefore examined the strength of the association between risk factors for thrombosis and BIH. Methods-The incidence of prothrombotic abnormalities among a mixed prospectively and retrospectively investigated cohort of 38 patients with BIH, was compared with healthy obese subjects, and patients with other neurological diseases. Prothrombotic abnormalities investigated included anticardiolipin antibodies, lupus anticoagulant, antithrombin III, proteins $C$ and $S$, plasma fibrinogen, kaolin cephalin clotting time, prothrombin time, and full blood counts.
\end{abstract}

Results-Evidence for the presence of an antiphospholipid antibody was found in $32 \%$ of cases. Cases of familial deficiency of antithrombin III, thrombocytosis, and polycythaemia were also noted. Additionally, an increased concentration of plasma fibrinogen was found in $26 \%$. A coagulation abnormality was more often detectable in those subjects with normal or low body mass index and in those tested within six months of onset.

Conclusion-There is a thrombotic pathogenesis in some cases of BIH.

Department of

Neurology

J Sussman

G A B Davies-Jones

Department of

Haematology, Royal

Hallamshire Hospital,

Glossop Road,

Sheffield S10 2JF, UK

$M$ Leach

R Malia

Department of

Medicine and

Therapeutics,

University of

Aberdeen, AB9 2ZD,

UK

M Greaves

Correspondence to: Dr J Sussman.

Received 27 June 1995

and in final revised form

19 August 1996

Accepted 16 September 1996
(F Neurol Neurosurg Psychiatry 1997;62:229-233)

Keywords: benign intracranial hypertension; pseudotumour cerebri; antiphospholipid antibodies

Benign intracranial hypertension (BIH) is a syndrome of raised intracranial pressure with headache and papilloedema, usually without localising signs, obstruction, or deformation of the ventricular system, in an alert and oriented patient, for which no cause can be found. ${ }^{1-3}$ It may result in serious sequelae; in a cumulative series of 1020 cases, $29 \%$ developed visual loss and $3 \%$ became blind. ${ }^{4}$ The risk of recurrence has been reported as $8 \%-12 \%$ over a decade. ${ }^{56}$

A proportion of patients, particularly those associated with chronic middle ear infection, arise from dural venous sinus thrombosis. ${ }^{7}$ In a series of 16 patients in which sinography was performed, five patients $(31 \%)$ had sinus occlusion, of whom only two had otitis. ${ }^{8} \mathrm{BIH}$ has also been described in association with polycythaemia rubra vera, ${ }^{9}$ essential thrombocythaemia, ${ }^{10}$ myeloma, ${ }^{11}$ and protein C deficiency, ${ }^{12}$ all conditions associated with a prothrombotic state. It is not known whether intracranial thrombosis was present in most of these cases.

Studies of the pathophysiology of BIH have identified an increase in resistance to CSF drainage at the level of the arachnoid villi, the exvaginations of the subarachnoid space into the large dural sinuses through which water from CSF is returned to the circulation. ${ }^{813}$ Even though conventional angiography has found venous sinus thrombosis in some cases, in others which were associated with a risk factor for thrombosis, none has been demonstrated. ${ }^{10}$ It is therefore possible that unrecognised non-occlusive thrombus lining dural vessels might impede CSF absorption. A predisposition to venous thrombosis is a feature of the familial thrombophilic states (deficiency of antithrombin, protein $C$, protein $S$ ), and the recently described resistance to activated protein $C$ associated with inheritance of factor V Leiden, and the antiphospholipid syndrome.$^{14}$ Furthermore, plasma fibrinogen is a major determinant of blood viscosity and hence flow, as well as being a cofactor in platelet aggregation and the substrate for thrombin. The aim of the study was therefore to seek these prothrombotic abnormalities of coagulation in subjects with $\mathrm{BIH}$.

\section{Patients and methods}

Ethics committee approval was obtained for the study. A manual search of inpatient discharge letters and outpatient clinic copy letters was made for the period covering 1987 to 1992 at the Royal Hallamshire Hospital, Sheffield. New patients presenting during the course of the study were also included. A total of 44 patients who fulfilled the modified Dandy criteria were identified, ${ }^{1}$ of whom 38 could be traced and who agreed to assist in the research. One patient had since died, but had been investigated at the time of presentation. Nine $(24 \%)$ were investigated prospectively, all within one month of clinical onset and the remaining 29 were studied between three months and 13 years after onset.

Two control groups were used. Sixteen asymptomatic healthy females were selected 
from hospital staff, matched for obesity and age. A second group of 18 age matched controls with other neurological diseases (OND) was selected from among inpatients on the surgical and medical neurology wards. All had recently presented with non-vascular disease. Those in both control groups were investigated in the same manner as the patients with BIH.

\section{LABORATORY METHODS}

Full blood counts were performed on a Coulter St autoanalyser. Clotting times (thrombin time, prothrombin time, and kaolin cephalin clotting time (KCCT)) were performed on citrated plasma by standard methods. Plasma fibrinogen was assayed by the method of Clauss. ${ }^{15}$ Antithrombin was measured by chromogenic assay (BioMerieux, Lyon, France). Protein C was measured by chromogenic assay after snake venom activation. Total and free protein $S$ were assayed by enzyme linked immunosorbent assay (ELISA) with a polyethylene glycol precipitation step. Lupus anticoagulant was sought using the KCCT and the dilute Russell's viper venom time (DRVVT) with a platelet neutralisation procedure as previously described. ${ }^{16}$ The reference range for the DRVVT ratio $(1 \cdot 0-1 \cdot 09)$

Table 1 Results of investigations on patients with $B I H$

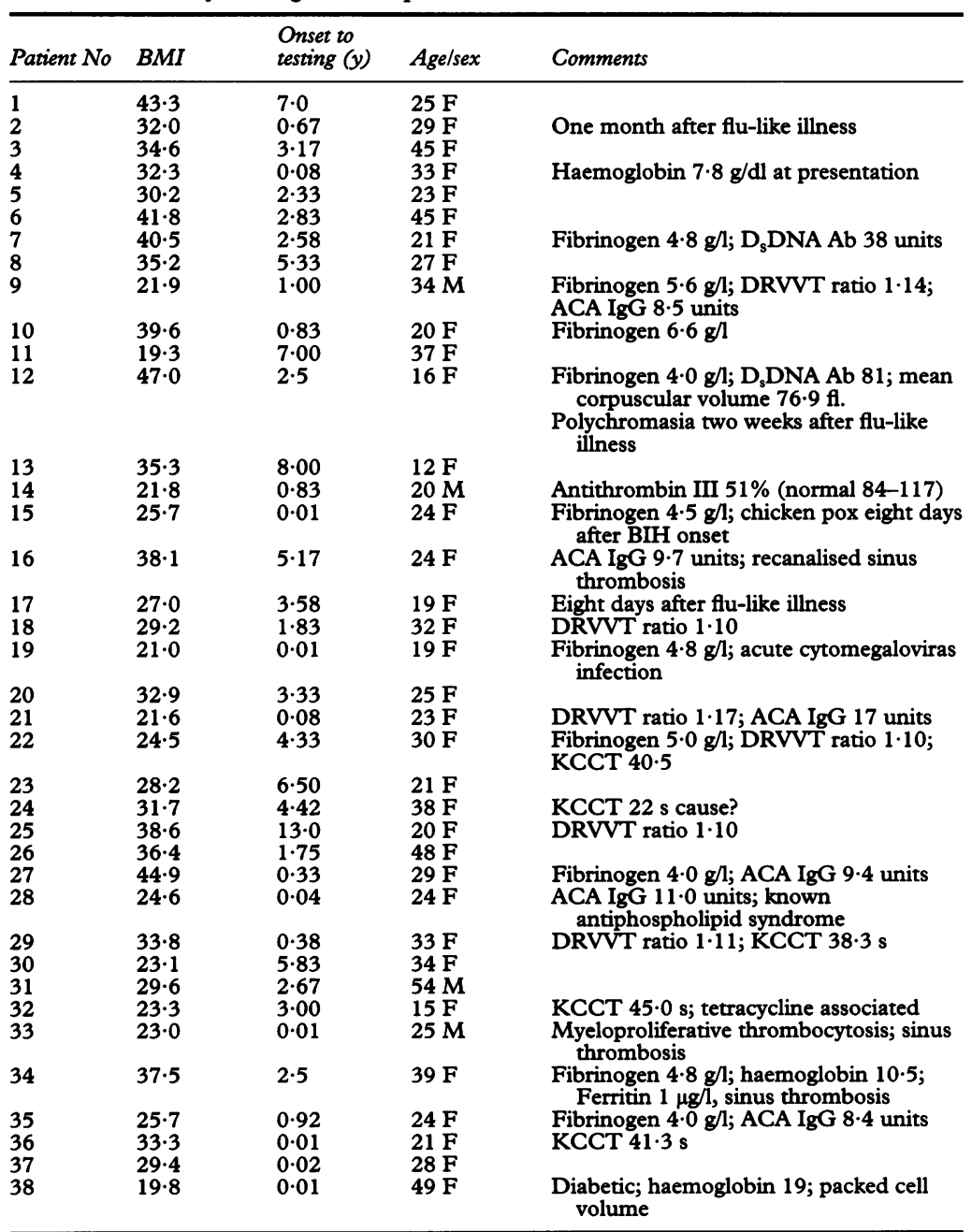

DRVVT = Dilute Russell's viper venom time; $A C A$ = anticardiolipin antibodies; $\mathrm{KCCT}=$ kaolin cephalin clotting time. was established on fresh plasma from 40 healthy subjects. Preanalytical variables were carefully standardised to maximise assay sensitivity, as previously recommended. ${ }^{17}{ }^{18}$ IgG and IgM anticardiolipin antibodies (ACAs) were assayed on serum using previously validated and standardised ELISAs. ${ }^{16}$ Because of the well recognised skewed distribution of ACA concentration the upper limit of normal was taken as the mean of the concentration of 40 healthy subjects $+3 \mathrm{SD}^{19}(5 \cdot 0$ and $8 \cdot 0$ $\mathrm{U} / \mathrm{ml}$ for IgM and IgG ACA respectively).

\section{Results}

The patients studied comprised 34 women and four men (ratio 8.5:1). The age at symptomatic onset was 28.6 (SD 9.9) (range 12-54) years. The association with obesity was confirmed (body mass index $31(7 \cdot 4)$, normal range 20-25), suggesting that this was a typical sample of patients with BIH.

Table 1 lists the findings in each patient. Abnormalities consistent with the presence of antiphospholipid antibodies (lupus anticoagulant or raised titre of anticardiolipin) were found in 11 patients (29\%). The DRVVT ratio was increased in six plasma samples $(1 \cdot 10-1 \cdot 17)$ and corrected in the platelet neutralisation procedure, a result highly consistent with the presence of a lupus anticoagulant. In addition, the KCCT, a less sensitive test, was also prolonged in one of these patients (22). Two of those with a lupus anticoagulant also had a raised titre of IgG ACA (8.5 and 17 units). Increased IgG ACA (9.4-11.0 units) was also detected in four other patients (16, $27,28,35$ ), one of whom, a 24 year old nonobese woman carried an established diagnosis of antiphospholipid syndrome based on a history of pulmonary embolism in the presence of previously identified ACA (28).

Mean plasma fibrinogen did not differ significantly between the BIH and the healthy obese control groups (3.26 (SD 1.12) v 2.98 (SD 0.8) $\mathrm{g} / \mathrm{l}$ ); however, the normal gaussian distribution seen among the controls was replaced by a distinct skew towards hyperfibrinogenaemia in the BIH group, with 10 patients $(26 \%)$ above the upper limit of the laboratory reference range (normal mean +2 $\mathrm{SD})$ of $3.9 \mathrm{~g} / 1$ (4.0-6.6 g/l; figure). Four of

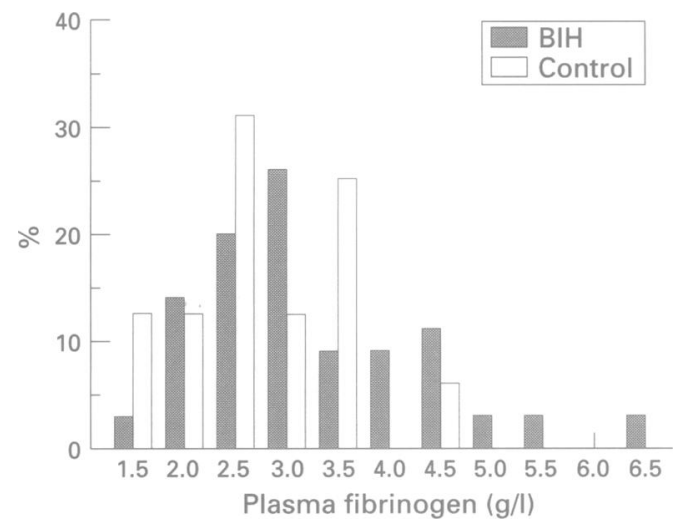

Fibrinogen level in patients with $\mathrm{BIH}$ and in healthy obese controls. 
Table 2 Results for patients and controls

\begin{tabular}{llllll}
\hline Patient group & All patients & BMI $<25$ & BMI $>25$ & Healthy obese & OND \\
\hline Number of subjects & 38 & 11 & 27 & 16 & 18 \\
APA & 12 & 5 & 7 & 1 & 3 \\
Hyperfibrinogenaemia & 10 & 3 & 7 & 1 & 3 \\
APA +hyperfibrinogenaemia & 4 & 2 & & \\
Antithrombin III deficiency & 1 & 1 & & \\
Myeloproliferative disease & 1 & 1 & & \\
Dehydrated diabetic & 1 & 1 & & \\
\hline
\end{tabular}

BMI = Body mass index; OND = other neurological diseases. APA = antiphospholipid antibody.

these had evidence of an antiphospholipid antibody in addition (patients $9,22,27,35$ ).

Abnormal fibrinogen and antiphospholipid antibody results were also found in the control groups. One subject in the obese control group had a raised titre of IgG ACA (10 units) together with a positive DRVVT; a second had a raised fibrinogen $(4.5 \mathrm{~g} / \mathrm{l})$. There was no significant relation between plasma fibrinogen concentration and body mass index among the controls. In the OND group, mean age was 30.4 (SD 5.6) years, but body mass index was 23.9 (SD 4.6). Antiphospholipid antibodies were found in three patients with measles encephalitis (DRVVT 1.15), Miller Fisher syndrome (DRVVT 1.1), and sciatica (DRVVT 1.14). The three patients with high fibrinogens had vestibular neuronitis $(4.4 \mathrm{~g} / 1)$, chronic subdural haematoma $(4 \cdot 2 \mathrm{~g} / 1)$, and encephalitis $(4.4 \mathrm{~g} / 1)$. The highest fibrinogen was $4.4 \mathrm{~g} / 1$, in comparison with the higher concentrations among patients with BIH (table 2).

Other highly prothrombotic disease associations were noted among the BIH group. One patient, a 20 year old man with a normal body mass index (patient 14), had reduced plasma antithrombin concentration, of $51 \%$ (normal 84-117). This persisted and further investigation disclosed type 1 familial antithrombin deficiency, present also in his mother. A 25 year old non-obese man (patient 33) presented with typical BIH. He was noted to have a platelet count of $530 \times 10^{9} /$. Further investigation including bone marrow aspiration showed a myeloproliferative disorder, and a right sigmoid sinus occlusion was indicated angiographically. A 49 year old non-obese diabetic woman (38) was admitted with diabetic ketoacidosis which had resulted in severe dehydration with a venous packed cell volume of $58 \%$. She was treated in the usual manner and made a full recovery. In retrospect she developed minor visual symptoms seven days after admission, and subsequently presented four weeks later with signs and symptoms of raised intracranial pressure. Other previously recognised associations were iron deficiency anaemia in three patients $(4,12,34)$ and tetracycline therapy in one patient (32) with a prolonged KCCT, not correctable by the addition of plasma, which persisted after resolution of all signs and symptoms. This could also represent a lupus anticoagulant.

Eleven of the 38 subjects had low or normal body mass index $(\leqslant 25 \cdot 0)$. Three of the four males were in this group. Nine of the 11 had a potential prothrombotic state (five with antiphospholipid antibody, of whom two also had hyperfibrinogenaemia, one with isolated hyperfibrinogenaemia, one with antithrombin deficiency, one with thrombocytosis, and one diabetic patient with dehydration (table 2 ).

There was no overall difference in obesity between those with and without a coagulation abnormality (body mass index 30.4 (SD 8) $v$ 31.9 (SD 6)), although the chance of an abnormality being found was greater in those with normal or low weight $(82 \% v 52 \%)$. In particular $45 \%$ of the non-obese in comparison with $26 \%$ of the obese had antiphospholipid antibodies (table 2). Body mass index was comparable between smokers $(n=16$, 30.6 (SD 7.4)) and non-smokers (30.9 (SD $7 \cdot 7)$ ). Surprisingly, there was no relation between plasma fibrinogen concentration and body mass index in patients or controls. Body mass index was comparable between those with a high fibrinogen who smoked $(30.0$ (SD 7.5)) and those who did not (35.6 (SD 10.4)). There was no significant difference in age between those with or without a haematological abnormality. Three of the nine women with a raised fibrinogen were using an oral contraceptive at presentation. In comparison, only one of the nine women with antiphospholipid antibodies was using an oral contraceptive.

Of the 13 patients investigated within six months of onset of symptoms, $12(92.3 \%)$ had abnormalities compared with $43 \%$ of those investigated more than six months from presentation, and $33 \%$ in the OND control group. More than 12 months had elapsed since the onset of symptoms in 21 of the patients. Eleven $(52 \%)$ had had at least one symptomatic relapse after what seemed to be remission from papilloedema and headache, and when measured, normalising of the CSF pressure. Neither body mass index nor the presence of a clotting abnormality was a predictor of the course of the disease.

Angiography was performed on 18 patients. Three showed evidence of dural sinus thrombosis, and each had abnormalities of coagulation (table 1, patients 16, 33, 34). Because angiography was not always performed in clinically typical cases it was not possible to quantify any association between abnormalities of coagulation and angiographic findings. The patient with antithrombin III deficiency (14) had a normal angiogram. Seven patients were investigated by cerebral angiography within one month of clinical onset. None of these had an angiographic abnormality, although six had clotting abnormalities (one hyperfibrinogenaemia, three antiphospholipid antibodies, one tetracycline induced with prolonged KCCT, one dehydrated diabetic). 


\section{Discussion}

The incidence of antiphospholipid antibody was $31 \%$ among the patients with $\mathrm{BIH}$, rising to $45 \%$ in those with normal or low body weight. This compares with $6 \%$ in the healthy obese, and $17 \%$ in the OND group. Mild to moderate hyperfibrinogenaemia was found in $27 \%$ of those with normal or low body weight, in comparison with $6 \%$ in the healthy obese control group, and $17 \%$ in the OND group. Ten per cent of those with normal or low body weight in the BIH group had antiphospholipid antibodies combined with hyperfibrinogenaemia. A further three patients $(27 \%)$ in the low to normal weight group had conditions closely associated with thrombosis; thrombocytosis, antithrombin III deficiency, and dehydration. This study illustrates the high frequency of abnormalities associated with intravascular thrombosis found in subjects with BIH. Clinical experience suggests that there is a greater probability that a cause will be found in those of normal weight. This is confirmed in this study in which $82 \%$ of those of normal or low weight had a potentially prothrombotic abnormality in comparison with $52 \%$ of the overweight patients (table 3).

An association between arterial and venous thromboembolic disease and the detection of antiphospholipid antibodies is accepted, although causality has not been shown. Recent findings suggest that plasma or IgG from antiphospholipid positive subjects can influence natural anticoagulant mechanisms and endothelial cell function in a manner which predisposes to thrombosis. This could be through the known interaction with phospholipid bound prothrombin, $\beta_{2}$-glycoprotein 1 , or protein C. An alternative explanation might be that antiphospholipid positivity is a surrogate marker for some other, possibly immunologically based, prothrombotic state. Although the ACA concentrations detected here were generally low, in those who were retested, persistence was demonstrated. The skewed distribution of the ACA concentration is well recognised, and the use of 3 SD from the mean as a limit of normality is accepted; sometimes less stringent measures of normality have been used. ${ }^{19}$ One patient (28) who had previously had pulmonary emboli, already fulfilled the criteria for the diagnosis of antiphospholipid syndrome, before her episode of BIH. A second patient (21) gave a history of two successive miscarriages, another an association with ACA. In a third subject, an obese woman (36), BIH developed against a background of neurosarcoidosis, positive tests for lupus anticoagulant, and livedo reticularis, which is a skin manifestation of the antiphospholipid syndrome. The six patients positive for lupus anticoagulant were identified using recommended laboratory procedures, and unlike

Table 3 Body mass index (BMI) and abnormal tests in patients with BIH

\begin{tabular}{lll}
\hline BMI & No of patients & Abnormal results (\%) \\
\hline $15-24 \cdot 9$ & 11 & $81 \cdot 8$ \\
$25-34 \cdot 9$ & 15 & $46 \cdot 7$ \\
$35 \cdot 0+$ & 12 & $58 \cdot 3$ \\
\hline
\end{tabular}

the situation with ACA, there is no evidence that the strength of lupus anticoagulant is predictive of disease, and a repeatedly positive result is potentiality of importance. As shown in the obese control group, an antiphospholipid antibody of no apparent relevance may occur in healthy subjects. In a study of 501 healthy pregnant women, $2 \cdot 8 \%$ were found to have an antiphospholipid antibody. ${ }^{16}$ An association between Guillain-Barré syndrome and encephalitis, and antiphospholipid antibodies, as noted in this group, has been previously described, ${ }^{20}$ and positive tests, which are often transient, may be associated with various infections.

The findings presented here suggest an association between antiphospholipid antibodies and BIH, supporting the concept of a thrombotic aetiology for some cases of the disorder. This is strongly corroborated by BIH as the presenting feature of familial antithrombin deficiency in one patient. Deficiency of antithrombin has an estimated prevalence of about 1 in 30000 of the population. ${ }^{17} \mathrm{~A}$ previously asymptomatic carrier has a $65 \%$ chance of a venous thrombotic event between the ages of 15 and 30 years. Finally, two other cases in this series were likely to have had a thrombotic pathogenesis - one after a period of severe dehydration with polycythaemia secondary to diabetic ketoacidosis, and the second with a thrombocytosis who was shown four years later, after a deterioration in his condition, to have a sigmoid sinus thrombosis on angiography.

In 10 patients the plasma fibrinogen was raised. Fibrinogen is a risk factor for venous thrombosis. ${ }^{21}$ It is an acute phase reactant and could, alternatively, merely be a marker for an underlying condition which consequently precipitates BIH. It is noteworthy that two patients with hyperfibrinogenaemia developed $\mathrm{BIH}$ within days of viral infection with chicken pox and with Cyclomegalovirus. The control group of patients with other neurological diseases further illustrates the effect of various conditions on fibrinogen. There is a recognised relation between plasma fibrinogen and obesity, as well as oral contraceptive use and smoking. 22-24 Fibrinogen, however, is a major determinant of the viscosity of plasma and therefore blood, and has a major role in platelet aggregation as well as acting as the substrate for fibrin formation. The presence of increased plasma fibrinogen in BIH thus lends further support to the possible thrombotic pathogenesis of this disorder.

No consensus has yet formed on which investigations are required to exclude a cause for raised intracranial hypertension in order to make a diagnosis of BIH. Historically angiography has often not been performed. Matters may also be complicated by the uncertainty as to whether a causative relation exists between an abnormality found on investigation and the raised intracranial pressure. In the case of pseudotumour cerebri appearing in the context of neurosarcoidosis and an antiphospholipid antibody for example, it may be impossible to determine whether the condition has developed secondarily to raised protein or cells in the CSF interfering with CSF absorption, or whether an 
antiphospholipid antibody induced by the sarcoidosis has precipitated the disease. Other problems of causation arise in the literature. A link with steroid use has often been cited ${ }^{25-29}$ although with a CSF pleocytosis in some of the cases. Many of those given steroids were in fact receiving treatment for systemic lupus erythematosus, so again, this association must be regarded as tentative. There may be value in regarding the apparently idiopathic cases as a special group, but the failure to recognise that such cases form only part of the range of causes of BIH may result in the exclusion from the diagnosis of cases which offer useful insights into its pathophysiology.

The likely lesion in BIH is a failure to absorb CSF through the arachnoid villi. The condition has been shown to occur in patients with raised CSF protein ${ }^{30}$ and in leukaemic infiltration of arachnoid granulations, ${ }^{31}$ and experimental models show that this occurs as a result of an inhibition of bulk CSF absorption at the arachnoid villi. ${ }^{32} \mathrm{BIH}$ is known to occur in vitamin $\mathrm{A}$ deficiency in infant humans. Rats fed a vitamin A deficient diet exhibit reversible raised intracranial pressure, ${ }^{33}$ and vitamin A deficient calves have arachnoid villi fibrosis. ${ }^{34}$

It thus seems likely that any interference with the structural or metabolic integrity of the arachnoid villi might give rise to defects in CSF absorption. Whereas we have suggested that local thrombosis could be pathogenic, there are alternative hypotheses to account for the physical obstruction of fluid movement across the villi. Antiphospholipid antibodies might be induced as an epiphenomenon arising from endothelial damage caused, for example, by cytotoxic antiendothelial antibodies, which could expose negatively charged membrane phospholipid, ${ }^{35}$ in which case slowing of CSF absorption may be the consequence of this endothelial injury.

Failure to recognise that $\mathrm{BIH}$ is heterogeneous in aetiology and prognostic terms will have contributed to our uncertainty as to how it should be managed. As the natural history of antiphospholipid antibodies is uncertain, and as fibrinogen is an acute phase reactant, a prospective study is required in which patients are tested as soon as possible after the onset of symptoms and on a continuing basis. It may then be possible to identify risk factors, some of which may be transient, for the subgroups which will go on to develop significant visual damage or a non-remitting course.

We suggest that all patients should undergo cerebral venous angiography and that screening for thrombophilia may be indicated. This screening could now include tests for factor $\mathrm{V}$ Leiden, as this point mutation in the gene for factor $V$ has recently been described as a highly prevalent risk factor for limb vein thrombosis. ${ }^{36}$ It may then be possible to identify people in whom an antithrombotic therapeutic strategy would be logical.

1 Hoffman HJ. How is pseudotumor cerebri diagnosed? Arch Neurol 1982;43:167-8.

2 Corbett J, Mehta MP. Cerebrospinal fluid pressure in normal obese subjects and patients with pseudotumor cerebri. Neurology 1983;33:1386-8.
3 Dandy WE. Intracranial pressure without brain tumor. Ann Surg 1937;106:492-513.

Wall $M$. Idiopathic intracranial hypertension. Neurol Clin 1991;9:73-95.

5 Corbett JJ, Savino PJ, Thompson S, et al. Visual loss in pseudotumor cerebri. Arch Neurol 1982;33:1386-8.

6 Boddie HG, Banna M, Bradley WG. Benign intracranial hypertension. Brain 1974;97:313-26.

7 Foley J. Benign forms of intracranial hypertension - "toxic" and "ototic" forms. Brain 1955;78:1-41.

8 Janny P, Chazal J, Colnet G, Irthum B, Georget A-M. Benign intracranial hypertension and disorders of CSF absorption. Surg Neurol 1980;15:168-74.

9 Melamed E, Rchmilewitz EA, Reches A, Lavy S. Aseptic cavernous sinus thrombosis after internal carotid arterial occlusion in polycythaenia vera. $f$ Neurol Neurosurg Psychiatry 1976;39:320-4.

10 Esack A, Thompson G, Burmester $H$. Benign intracranial hypertension and essential thrombocythaemia. $\mathcal{F}$ Neurol Neurosurg Psychiatry 1989;52:914.

11 Wasan H, Mansi JL, Benjamin S, Powles R, Cunningham $D$. Myeloma and benign intracranial hypertension. $B M \mathcal{F}$ 1992;304:685.

12 Massons J, Arboix A, Oliveres M, Besses C, Munoz C, Titus F. Cerebral venous thrombosis and hereditary protein C deficiency. Neurologia 1992;7:34-8.

13 Johnston I. Reduced CSF absorption syndrome. Reappraisal of benign intracranial hypertension and related conditions. Lancet 1973;ii:418-21.

14 Greaves M, Davies-Jones GAB. Neurological abnormalities associated with coagulopathies. Elsevier, 1993.

15 Clauss A. Rapid physiological coagulation method in determination of fibrinogen. Acta Haematologica 1957;17 237-46.

16 Creagh MD, Maglia RG, Cooper SM, Smith AR, Duncan SLB, Greaves M. Screening for the lupus anticoagulan and anticardiolipin antibody in women with fetal loss. $\mathcal{F}$ Clin Pathol 1991;44:45-7.

17 Greaves M, Preston FE. Clinical and laboratory aspects of thrombophilia, in Recent advances in Blood Coagulation 1993. London: Churchill Livingstone, 119-40.

18 Machin SJ, Giddings J, Greaves M, Hutton R. Detection of the lupus-like anticoagulant: current laboratory practice in the United Kingdom. $\mathcal{F}$ Clin Pathol 1990;43:73-9.

19 Harris EN, Gharavie AE, Hegde U, et al. Anticardiolpin antibodies in autoimmune thrombocytopaenic purpura. Br F Haematol 1985;59:231-4.

20 Levine SR, Welch KMA. Spectrum of neurological diseases associated with antiphospholipid antibody. Arch Neurol 1987;44:876-83.

21 Koster T, Rosendaal FR, Reitsma PH, et al. Factor VII and fibrinogen levels as risk factors for venous thrombosiscase control study of plasma levels and DNA polymorphism. Thrombos Hamostas 1994;71:719-22.

22 Ogston D, McAndrew GM. Fibrinolysis in obesity. Lancet 1974;2:1205-7.

23 Meade TW, Chakrabarti R, Haines AP, North WRS, Stirling Y. Characteristics affecting fibrinolytic activity
and plasma fibrinogen concentrations. $B M \mathcal{F} 1979 ; 1$ : and plasn

24 Balleisen L, Bailey J, Epping PH, Schulte H, Van de Loo J. Epidemiological study on factor VIII and fibrinogen in an industrial population. I: borderline data on the relation to age, body-weight, smoking, alcohol, pill-using and menopause. Thrombos Haemostas 1985;54:475-9.

25 Vyas CK, Talwar KK, Bhatnagar V, et al. Steroid-induced benign intracranial hypertension. Postgrad Med $\mathcal{F} 1981$; 57:181-2.

26 Greer M. Benign intracranial hypertension: II following corticosteroid therapy. Neurology 1963;13:439-41.

27 Neville BGR, Wilson J. Benign intracranial hypertension following corticosteroid withdrawal in children. $B M \mathcal{F}$ following cortico

28 Digre KB, Corbett J. Pseudotumor cerebri in men. Arch Neurol 1988;45:866-72.

29 Griffin JP. A review of the literature on benign intracranial hypertension associated with medication. Adverse Drug React Toxicol Rev 1992;11:41-58.

30 Risdale L, Moseley I. Thoracolumbar intraspinal tumours. Presenting features of raised intracranial pressure. $f$ Neurol Neurosurg Psychiatry 1978;41:737-45.

31 Pierro L, Brancato R, Zaganelli E, Guarisco L, Lazetta P. Ocular involvement in acute lymphoblastic leukaemia: an echographic study. Int Ophthalmol 1992;16:159-62.

32 Prockop LD, Fishman RA. Experimental pneumococcal meningitis: permiability changes influencing the concenmeningitis: permiability changes influencing the concentration of sugars and macromolecul
fluid. Arch Neurol 1968;19:449-63.

33 Karsarkis EJ, Maffea CJ, Johnson RN, Butler AB, Bass $\mathrm{NH}$. Intracranial hypertension in vitamin A deficient rats: reversible metabolic derangement of cerebrospinal fluid absorption. Neurology 1978;28:349.

34 Hayes KC. The fine structure of vitamin A deficiency II: arachnoid granulations and CSF pressure. Brain 1971; 94:213-24.

35 Hill MB, Phipps JL, Malia RG, et al. Characterisation and specificity of anti-endothelial cell membrane antibodies and their relationship to thrombosis in primary antiphospholipid syndrome (APS). Clin Exp Immunol 1995; 102:368-72.

36 Svensson PGA, Dahlback B. Resistance to activated protein $\mathrm{C}$ as a basis for venous thrombosis. $N$ Engl $f \mathrm{Med}$ 1994;330:517-22. 